\title{
UNIVERSAL EQUIVARIANT BUNDLES
}

\author{
MITUTAKA MURAYAMA AND KAZUHISA SHIMAKAWA
}

(Communicated by Thomas Goodwillie)

\begin{abstract}
We present a method of functorially constructing universal equivariant bundles.
\end{abstract}

\section{INTRODUCTION}

Let $G$ and $A$ be topological groups, and suppose $G$ acts on $A$ via a continuous homomorphism $\alpha: G \rightarrow$ Aut $A$. In [16], tom Dieck introduced a notion of $(G, \alpha, A)$-bundles which includes the notions of usual $G$-equivariant bundles [13], [18], [2], [5] (the case $\alpha$ is trivial) and Atiyah's vector bundles with Reality [1] ( $\alpha$ is the complex conjugation $\mathbf{Z} / 2 \mathbf{Z} \rightarrow \operatorname{Aut} U(n))$. He proved the existence of a universal $(G, \alpha, A)$-bundle in the case $G$ is a compact Lie group and $A$ is an inductive limit of Lie groups with finitely many connected components. However, his construction as well as other constructions involving choice of representatives for equivalence classes of local objects (e.g., [17], [5]) is far from being functorial in $G$ and $A$.

In this article, we present a new construction of universal $(G, \alpha, A)$-bundles under the assumption that $G$ is a compact Lie group or a discrete group and $A$ is any topological group. It has three advantages: it is quite explicit, does not use the (generally unknown) classification of local objects, and, especially, is functorial in $(G, \alpha, A)$, the meaning of which will be made clear in due course. Our method is a generalization of the functor category approach used in $[15, \S 3]$ and is closely connected with the mapping space approach used by May [8] (cf. the final remark in $\S 3$ ).

\section{Numerable $(G, \alpha, A)$-BundLes}

In this section we recall the definition and basic properties of $(G, \alpha, A)$ bundles. We write $g a=\alpha(g, a)$ for $g \in G$ and $a \in A$.

Definition 1.1. A principal $(G, \alpha, A)$-bundle is a locally trivial principal $A$ bundle $p: E \rightarrow B$ enjoying the following properties:

(1) Both $E$ and $B$ are left $G$-spaces, and $p$ is $G$-equivariant.

(2) For every $g \in G, a \in A$, and $e \in E$, we have $g(e \cdot a)=g e \cdot g a$.

Received by the editors February 16, 1993 and, in revised form, July 20, 1993.

1991 Mathematics Subject Classification. Primary 55R91. 
The latter property is equivalent to saying that the semi-direct product $G \times_{\alpha}$ $A$, the product $G \times A$ viewed as a topological group under the multiplication

$$
(g, a)\left(g^{\prime}, a^{\prime}\right)=\left(g g^{\prime}, g a^{\prime} \cdot a\right),
$$

acts on the left of $E$ by $(g, a) e=g e \cdot a,(g, a) \in G \times{ }_{\alpha} A$.

There are evident notions of $(G, \alpha, A)$-bundle maps and induced bundles. If $F$ admits left $G$ and $A$ actions satisfying $g(a \cdot x)=g a \cdot g x$ then we can form the associated $(G, \alpha, A)$-bundle $E \times{ }_{A} F \rightarrow B$ in such a way that the (diagonal) $G$-action on the total space is compatible with the $A$-actions on its fibres.

Definition 1.2. For any closed subgroup $H$ of $G$, a principal $(G, \alpha, A)$-bundle over the homogeneous space $G / H$ is called a local object.

A continuous map $\phi: H \rightarrow A$ is called a crossed homomorphism if $\phi(h k)=$ $\phi(h) \cdot h \phi(k)$ holds for $h, k \in H$. Let $p: E \rightarrow G / H$ be a local object and let $e \in p^{-1}(H)$. Then the equation $h e=e \cdot \phi(h)$ determines a crossed homomorphism $\phi: H \rightarrow A$, and the isotropy group of $e$ under the $G \times_{\alpha} A$-action on $E$ is given as the graph of the map $h \mapsto \phi(h)^{-1}$. Since $G \times_{\alpha} A$ acts transitively on $E$, there is a $G \times{ }_{\alpha} A$-equivariant homeomorphism of $E$ with $G \times{ }_{\alpha} A / H$, the orbit space of $G \times{ }_{\alpha} A$ with respect to the right $H$-action

$$
(g, a) \mapsto(g, a)\left(h, \phi(h)^{-1}\right)=\left(g h, g \phi(h)^{-1} \cdot a\right), \quad h \in H .
$$

We have shown:

Lemma 1.3. For every local object $p: E \rightarrow G / H$, there exist a crossed homomorphism $\phi: H \rightarrow A$ and $a(G, \alpha, A)$-bundle equivalence $E \cong G \times_{\alpha} A / H$ over $G / H$.

More generally, a $(G, \alpha, A)$-bundle with fibre $F$ is called trivial if it is equivalent to a $(G, \alpha, A)$-bundle of the form

$$
\left(G \times_{\alpha} F\right) \times_{H} V \rightarrow G \times_{H} V,
$$

where $V$ is a left $H$-space and $H$ acts on the right of $G \times{ }_{\alpha} F=G \times F$ by $(g, f) \mapsto\left(g h, g \phi(h)^{-1} \cdot f\right), \phi$ a crossed homomorphism $H \rightarrow A$. Clearly, a principal $(G, \alpha, A)$-bundle is trivial if and only if there is a $(G, \alpha, A)$-bundle map to a local object.

Definition 1.4. A $(G, \alpha, A)$-bundle $p: E \rightarrow B$ is called locally trivial if there is an open cover $\left\{V_{\beta}\right\}$ of $B$ such that for each $\beta$,

(1) $V_{\beta}$ is $G$-invariant, and

(2) $p^{-1}\left(V_{\beta}\right) \rightarrow V_{\beta}$ is a trivial $(G, \alpha, A)$-bundle.

If, moreover, there exists a subordinate partition of unity $\left\{\lambda_{\beta}\right\}$ such that each $\lambda_{\beta}$ is $G$-invariant then $p$ is called a numerable $(G, \alpha, A)$-bundle.

If $G$ and $A$ are compact Lie groups then every $(G, \alpha, A)$-bundle is locally trivial if its base space $B$ is completely regular and is numerable if $B$ is paracompact. (See [5, Corollary 1.5] for an argument using the existence of a slice [3].) In general, local triviality restricts the type of $G$-action on the base space. 


\section{Classification of equivariant Bundles}

A numerable principal $(G, \alpha, A)$-bundle $p: E \rightarrow B$ is called universal if for any $G$-space $X$, the equivalence classes of numerable $(G, \alpha, A)$-bundles over $X$ are in bijective correspondence with the equivariant homotopy classes of $G$ maps of $X$ into $B$ under the correspondence given by induced bundles. The goal of this section is to prove the following. (Compare [16, Satz 5.1] and [5, Theorem 2.14].)

Theorem 2.1. Let $p: E \rightarrow B$ be a numerable principal $(G, \alpha, A)$-bundle. Then $p$ is universal if and only if for every closed subgroup $H$ and every crossed homomorphism $\phi: H \rightarrow A, E$ is $H$-contractible under the $H$-action $e \mapsto$ he $\phi(h)^{-1}, h \in H$.

Write $G$-SEP and $G$-CHP for $G$-section extension property and $G$-covering homotopy property respectively. Arguing as in [5, Proposition 2.3, Corollaries 2.2 and 2.9], we have following three lemmas.

Lemma 2.2. Let $p: E \rightarrow B$ be a $G$-map and $\left\{V_{\beta}\right\}$ be a numerable $G$-cover of $B$. If $p$ has the G-SEP over each $V_{\beta}$ then $p$ has the G-SEP.

Lemma 2.3. Let $H$ be a closed subgroup of $G$. Let $V$ and $F$ be $H$-spaces and give $V \times F$ the diagonal $H$-action. If $F$ is $H$-contractible, the projection $p: G \times_{H}(V \times F) \rightarrow G \times_{H} V$ has the $G-S E P$.

Lemma 2.4. Let $G$ be a compact Lie group or a discrete group, and let $X$ be a $G$-space. If $E$ is a trivial $(G, \alpha, A)$-bundle over $X \times I$ then $E \cong(E \mid X) \times I$, hence has the G-CHP.

As a consequence of Lemma 2.4 we have:

Corollary 2.5. A numerable $(G, \alpha, A)$-bundle has the $G$-CHP.

We are now ready to prove Theorem 2.1. Assume that $p: E \rightarrow B$ satisfies the condition of the theorem. By Corollary 2.5, we get a natural map

$$
\gamma:[X, B]^{G} \rightarrow\{\text { Equivalence classes of }(G, \alpha, A) \text {-bundles over } X\}
$$

which assigns to each $f: X \rightarrow B$ the induced bundle $f^{*} p: f^{*} E \rightarrow X$.

We first show that $\gamma$ is surjective. Given a numerable principal $(G, \alpha, A)$ bundle $q: D \rightarrow X$, let $\langle q, p\rangle:\langle D, E\rangle \rightarrow X$ be the 'functional bundle' [4, 7.5] whose fibre on $x \in X$ consists of all admissible maps of $D_{x}$ into $E$. Under the correspondence

$$
\left(f: D_{x} \rightarrow E\right) \mapsto[d, f(d)] \in\left(D_{x} \times E\right) / A, \quad d \in D_{x},
$$

$\langle D, E\rangle$ is naturally isomorphic to the orbit space $(D \times E) / A$. In other words, $\langle q, p\rangle$ is the associated bundle of $D$ with fibre $E$, where $A$ acts on the left of $E$ by $a \mapsto e \cdot a^{-1}, a \in A$. Since $q$ is numerable, $\langle q, p\rangle$ is a numerable $(G, \alpha, A)$-bundle.

By the definition, a section in $\langle q, p\rangle$ over $U \subset X$ is the same as a bundle map $q \mid U \rightarrow p$. Thus, to prove the surjectivity of $\gamma$, we need only show that $\langle q, p\rangle$ has the $G$-SEP. Moreover, by Lemma 2.2 we may assume that $q$ is a trivial bundle $\left(G \times{ }_{\alpha} A\right) \times_{H} V \rightarrow G \times_{H} V$. 
Now the total space of $\langle q, p\rangle$ can be identified with $G \times_{H}(V \times E)$, where $H$ acts on $E$ by $(h, e) \mapsto h e \cdot \phi(h)^{-1}$ and diagonally on $V \times E$, through a bundle isomorphism

$$
\begin{gathered}
\left(\left(G \times_{\alpha} A\right) \times_{H} V\right) \times_{A} E \rightarrow G \times_{H}(V \times E), \\
{[[g, a, v], e] \mapsto\left[g, v, g^{-1}\left(e \cdot a^{-1}\right)\right] .}
\end{gathered}
$$

By Lemma 2.3, we see that $\langle q, p\rangle$ has the G-SEP.

The injectivity of $\gamma$ is proved by the similar argument with $X$ replaced by $X \times I$. Thus $p: E \rightarrow B$ is a universal $(G, \alpha, A)$-bundle.

Conversely, assume that $p: E \rightarrow B$ is a universal $(G, \alpha, A)$-bundle. As we shall see in the next section, there exists a $(G, \alpha, A)$-bundle which satisfies the condition of the theorem, and so, is universal. Since any two universal bundles are equivalent, we conclude that $p$ satisfies the condition of the theorem.

\section{FUNCTOR CATEGORY MODEL FOR UNIVERSAL EQUIVARIANT BUNDLES}

We present here a functorial construction of universal $(G, \alpha, A)$-bundles by generalizing the method of $[15, \S 3]$.

Let $p: U A \rightarrow B A$ be a universal $A$-bundle. By using a functorial construction of universal bundles (e.g., [11], [10], [12], [7]), we may assume that $p$ is a $(G, \alpha, A)$-bundle with respect to the $G$-action induced by $\alpha$.

Let $\mathscr{G} A$ be a topological category with

$$
\operatorname{ob} \mathscr{G} A=B A, \quad \operatorname{mor} \mathscr{G} A=\langle U A, U A\rangle=(U A \times U A) / A,
$$

where $[b, a] \in\langle U A, U A\rangle$ is regarded as an arrow from $p(a)$ to $p(b)$, and with composition law $[c, b] \circ[b, a]=[c, a]$. (With suitable choice of representatives, every composable pair of arrows in $\mathscr{G} A$ is of the form $([c, b],[b, a])$.)

Similarly, let $\mathscr{S} A$ be a topological category with

$$
\text { ob } \mathscr{S} A=U A, \quad \operatorname{mor} \mathscr{S} A=\langle U A, U A\rangle \times_{B A} U A,
$$

where $([b, a], a) \in\langle U A, U A\rangle \times_{B A} U A$ is regarded as an arrow from $a$ to $b$, and with composition law induced by that of $\mathscr{G} A$.

$\mathscr{S} A$ is fibred over $\mathscr{G} A$ by a functor $\pi: \mathscr{S}_{A} \rightarrow B A$ induced by $p$. Moreover, under the natural isomorphism $U A \times U A \cong\langle U A, U A\rangle \times_{B A} U A,(b, a) \mapsto$ $([b, a], a), \mathscr{S} A$ is identified with the category with a unique isomorphism between each pair of elements of $U A$. Hence $\mathscr{S}_{A}$ is equivalent to the trivial category with one object and one morphism.

Denote by $E G$ the translation category of $G$, that is, a contractible category with ob $E G=G$ and $\operatorname{mor} E G=G \times G$, where $(y, x) \in G \times G$ is viewed as a unique isomorphism $x \rightarrow y$. Let $\operatorname{Cat}(E G, \mathscr{S} A)$ be the category of continuous functors and continuous natural transformations of $E G$ into $\mathscr{S} A$. Since $\mathscr{S} A$ has a unique isomorphism between each pair of its objects, a functor $f: E G \rightarrow$ $\mathscr{S} A$ is completely determined by its restriction to objects, ob $f: G \rightarrow U A$. Thus the nerve of the contractible category $\operatorname{Cat}(E G, \mathscr{S} A)$ is given by

$$
N_{k} \operatorname{Cat}(E G, \mathscr{S} A)=\operatorname{Map}(G, U A)^{k+1}, \quad k \geq 0,
$$


where $\operatorname{Map}(G, U A)$ is the set of continuous maps of $G$ into $U A$, together with face and degeneracy operators induced by projections and diagonal embeddings respectively.

Let $G \times{ }_{\alpha} A$ act on $\operatorname{Cat}(E G, \mathscr{S} A)$ via its action on $\operatorname{Map}(G, U A)$,

$$
(g, a) f(x)=g f(x g) \cdot a, \quad(g, a) \in G \times \times_{\alpha} A .
$$

We topologize $\operatorname{Cat}(E G, \mathscr{S} A)$ in two ways. The strong topology is obtained by imposing on each $N_{k} \operatorname{Cat}(E G, \mathscr{S} A)$ the strongest topology such that its $G \times{ }_{\alpha} A$-action is continuous. On the other hand, the compact-open topology on $\operatorname{Map}(G, U A)$ (retopologized, if necessary, so as to be compactly generated [9, $\S 2])$ gives rise to another topology on $\operatorname{Cat}(E G, \mathscr{S} A)$, called natural topology. With respect to either topology, $G \times_{\alpha} A$ acts continuously on $\operatorname{Cat}(E G, \mathscr{S} A)$ and the functor

$$
\eta: \operatorname{Cat}(E G, \mathscr{S} A) \rightarrow \operatorname{Cat}(E G, \mathscr{G} A)
$$

induced by $\pi: \mathscr{S} A \rightarrow \mathscr{S}_{A}$ is a $G$-equivariant continuous functor. In fact, $N_{k} \eta$ is the natural map of $\operatorname{Map}(G, U A)^{k+1}$ onto the orbit space

$$
N_{k} \operatorname{Cat}(E G, \mathscr{G} A)=\operatorname{Map}(G, U A)^{k+1} / A, \quad k \geq 0,
$$

with respect to the diagonal $A$-action.

Recall from [14, Appendix A] that (the nerve of) a topological category $C$ has the naive realization $|C|$ and also the fat realization $\|C\|$. With the strong topology on $\operatorname{Cat}(E G, \mathscr{S} A)$, we will prove:

Theorem 3.1.

$$
\|\eta\|:\|\operatorname{Cat}(E G, \mathscr{S} A)\| \rightarrow\|\operatorname{Cat}(E G, \mathscr{G} A)\|
$$

is a universal $(G, \alpha, A)$-bundle.

Remark. It is desirable if we can prove Theorem 3.1 with the natural topology on $\operatorname{Cat}(E G, \mathscr{S} A)$ and/or with the naive realization instead of the fat one. This is certainly the case if both $G$ and $A$ are compact Lie groups.

Before proving the theorem, let us examine the functorial property of $\|\eta\|$ with respect to $(G, \alpha, A)$.

A morphism of $(G, \alpha, A)$ to another such system $(K, \gamma, C)$ is a pair of homomorphisms $l: K \rightarrow G$ and $r: A \rightarrow C$ such that $r(l(k) a)=k r(a)$ holds for $k \in K, a \in A$. Such a morphism $\mu=(l, r)$ functorially gives rise to a commutative square

$$
\begin{array}{cc}
\|\operatorname{Cat}(E G, \mathscr{S} A)\| \stackrel{U \mu}{\longrightarrow} & \|\operatorname{Cat}(E K, \mathscr{S} C)\| \\
\downarrow & \downarrow \eta \\
\|\operatorname{Cat}(E G, \mathscr{G} A)\| \underset{B \mu}{\stackrel{\longrightarrow}{\longrightarrow}}\|\operatorname{Cat}(E K, \mathscr{G} C)\|
\end{array}
$$

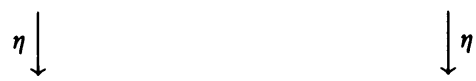

where $U \mu$ and $B \mu$ are induced by the functors $E K \rightarrow E G$ and $U A \rightarrow U C$ associated with $l$ and $r$ respectively. 
Let $A$ act on $C$ via $r$ and let $K \times_{\gamma} C$ act on $\|\operatorname{Cat}(E G, \mathscr{S} A)\| \times_{A} C$ by $(k, c)[e, d]=[l(k) e, k d \cdot c],(k, c) \in K \times{ }_{\gamma} C$. Then

$$
\|\operatorname{Cat}(E G, \mathscr{S} A)\| \times_{A} C \rightarrow\|\operatorname{Cat}(E K, \mathscr{S} C)\|, \quad[e, c] \mapsto U \mu(e) \cdot c,
$$

is a $(K, \gamma, C)$-bundle map over $B \mu$ or, equivalently, $B \mu$ is a classifying map for the associated bundle $\|\operatorname{Cat}(E G, \mathscr{S} A)\| \times_{A} C \rightarrow\|\operatorname{Cat}(E G, \mathscr{G} A)\|$ regarded as a principal $(K, \gamma, C)$-bundle. In particular, if $l$ is the inclusion of a closed subgroup $K$ of $G$ and $r$ the identity of $A$ then $B \mu$ corresponds to the restriction of the group action from $G$ to $K$ and, hence, is a $K$-equivalence. On the other hand, if $l$ is the identity and $r$ the inclusion of a closed subgroup $A$ of $C$ then $B \mu$ corresponds to the reduction of the structure groups (in the equivariant setting).

To prove Theorem 3.1, it suffices to show the following two lemmas.

Lemma 3.2. $\|\eta\|$ is a numerable principal $(G, \alpha, A)$-bundle.

Lemma 3.3. For any crossed homomorphism $\phi: H \rightarrow A,\|\operatorname{Cat}(E G, \mathscr{S} A)\|$ is $H$-contractible under the $H$-action $e \mapsto h e \cdot \phi(h)^{-1}, h \in H$.

Lemma 3.2 is proved by the argument of $[4, \S 8]$ together with the fact that

$$
N_{k} \eta: N_{k} \operatorname{Cat}(E G, \mathscr{S} A) \rightarrow N_{k} \operatorname{Cat}(E G, \mathscr{G} A)
$$

is a disjoint union of local objects, as follows by the property of the strong topology.

We now prove Lemma 3.3. In fact, we will show that $\operatorname{Cat}(E G, \mathscr{S} A)$ has an $H$-fixed terminal object, implying both the naive and the fat realizations of $\operatorname{Cat}(E G, \mathscr{S} A)$ are $H$-contractible with respect to any topology such that the $G \times{ }_{\alpha} A$-action is continuous. (See [10, Proposition 1.2] for the construction of $H$-contraction.)

Let $q: G \times{ }_{\alpha} A / H \rightarrow G / H$ be the local object corresponding to a crossed homomorphism $\phi$. Since $p: U A \rightarrow B A$ is universal, there exists an $A$-bundle map $F: G \times{ }_{\alpha} A / H \rightarrow U A$. Define an object $f \in \operatorname{obCat}(E G, \mathscr{S} A)=\operatorname{Map}(G, U A)$ by

$$
f(x)=x^{-1} F([x, 1]) \in U A, \quad x \in G .
$$

Then one easily checks that $\left(h f \cdot \phi(h)^{-1}\right)(x)=f(x)$ holds for any $h \in H$ and $x \in G$. Thus $f$ is fixed by the $H$-action $e \mapsto h e \cdot \phi(h)^{-1}$ on $\operatorname{Cat}(E G, \mathscr{S} A)$.

Since there exists a unique isomorphism $f^{\prime} \rightarrow f$ for any object $f^{\prime}$ in $\operatorname{Cat}(E G, \mathscr{S} A), f$ is an $H$-fixed terminal (and initial) object. This proves Lemma 3.3.

Notice that in the argument above, we only used the property that $p: U A \rightarrow$ $B A$ classifies principal $A$-bundles over homogeneous spaces $G / H$. In particular, if $G$ is finite, we can take as $p$ the trivial bundle $A \rightarrow *$. In this case, $\mathscr{S} A$ is the translation category $E A$ of $A$ and $\mathscr{G} A$ is the group $A$ regarded as a category with one object. Since $\operatorname{Map}(G, A)$ is a product of copies of $A$, the nerve of $\operatorname{Cat}(E G, \mathscr{G} A)$ as well as that of $\operatorname{Cat}(E G, \mathscr{S} A)$ has particularly simple structure and can be regarded as an equivariant generalization of the geometric bar construction [10], [7]. 
Remark. May showed in [8] that if $G$ and $A$ are compact Lie groups then the natural map

$$
\operatorname{Map}(U G, U A) \rightarrow \operatorname{Map}(U G, U A) / A
$$

is a model for universal $(G, \alpha, A)$-bundle. This mapping space model is connected with our functor category model through a $G \times{ }_{\alpha} A$-equivalence

$$
\begin{aligned}
|\operatorname{Cat}(E G, \mathscr{S} A)| & \cong\left|\operatorname{Map}_{\bullet}\left(N_{\bullet} E G, N_{\bullet} \mathscr{S} A\right)\right| \rightarrow \operatorname{Map}(|E G|,|\mathscr{S} A|) \\
& \simeq \operatorname{Map}(U G, U A),
\end{aligned}
$$

where $\operatorname{Map}_{\bullet}(-,-)$ denotes the simplicial mapping space. We hope to discuss elsewhere a further generalization of our functor category model to the case of generalized equivariant bundles introduced by [6] and its connection with the corresponding generalization of the mapping space model [8].

\section{REFERENCES}

1. M. F. Atiyah, $K$-theory and reality, Quart. J. Math. Oxford Ser. (2) 17 (1966), 367-386.

2. E. Bierstone, The equivariant covering homotopy property for differentiable $G$-fibre bundles, J. Differential Geom. 8 (1973), 615-622.

3. G. Bredon, Introduction to compact transformation groups, Pure Appl. Math., vol. 46, Academic Press, New York, 1972.

4. A. Dold, Partitions of unity in the theory of fibrations, Ann. of Math. (2) 78 (1963), 223-255.

5. R. K. Lashof, Equivariant bundles, Illinois J. Math. 26 (1982), 257-271.

6. R. K. Lashof and J. P. May, Generalized equivariant bundles, Bull. Soc. Math. Belg. Sér. B 38 (1986), 265-271.

7. J. P. May, Classifying spaces and fibrations, Mem. Amer. Math. Soc., vol. 155, Amer. Math. Soc., Providence, RI, 1975.

8. Some remarks on equivariant bundles and classifying spaces, Astérisque 191 (1990), 239-253.

9. M. C. McCord, Classifying spaces and infinite symmetric products, Trans. Amer. Math. Soc. 146 (1969), 273-298.

10. R Milgram, The bar construction and abelian $H$-spaces, Illnois J. Math. 11 (1967), 242-250.

11. J. W. Milnor, Construction of universal bundles. II, Ann. of Math. (2) 63 (1956), 430-436.

12. G. Segal, Classifying spaces and spectral sequences, Inst. Hautes Études. Sci. Publ. Math. 34 (1968), 105-112.

13. —_ Equivariant K-theory, Inst. Hautes Études. Sci. Publ. Math. 34 (1968), 129-151.

14. _ Categories and cohomology theories, Topology 13 (1974), 293-312.

15. K. Shimakawa, Infinite loop $G$-spaces associated to monoidal $G$-graded categories, Publ. Res. Inst. Math. Sci. 25 (1989), 239-262.

16. T. tom Dieck, Faserbündel mit gruppenoperation, Arch. Math. 20 (1969), 136-143.

17. S. Waner, Equivariant classifying spaces and fibrations, Trans. Amer. Math. Soc. 258 (1980), 385-405.

18. S. Wasserman, Equivariant differential topology, Topology 8 (1969), 127-150.

Department of Mathematics, Tokyo Institute of Technology, O-Okayama, MeguroKU, TOKYO, JAPAN

E-mail address: murayama@math.titech.ac.jp

Department of Mathematics, Okayama University, Okayama 700, Japan

E-mail address: yfae08660ccews2.cc.okayama-u.ac.jp 